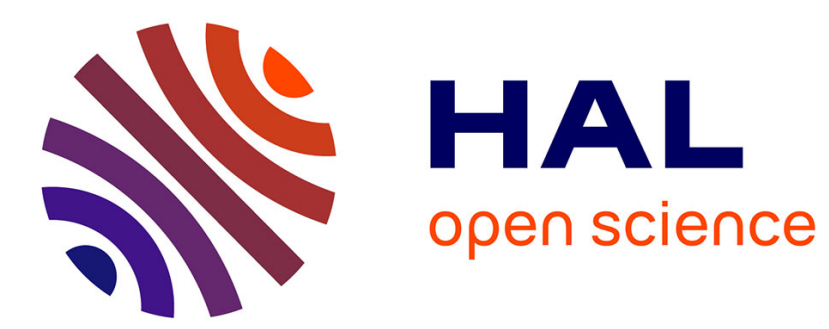

\title{
LE DROIT FRANÇAIS DES CONCOURS ENTRE PERMANENCE ET ÉVOLUTION
}

\author{
Delphine Espagno-Abadie
}

\section{To cite this version:}

Delphine Espagno-Abadie. LE DROIT FRANÇAIS DES CONCOURS ENTRE PERMANENCE ET ÉVOLUTION. Revue française d'administration publique, 2012. hal-03207233

\section{HAL Id: hal-03207233 \\ https://hal.science/hal-03207233}

Submitted on 24 Apr 2021

HAL is a multi-disciplinary open access archive for the deposit and dissemination of scientific research documents, whether they are published or not. The documents may come from teaching and research institutions in France or abroad, or from public or private research centers.
L'archive ouverte pluridisciplinaire HAL, est destinée au dépôt et à la diffusion de documents scientifiques de niveau recherche, publiés ou non, émanant des établissements d'enseignement et de recherche français ou étrangers, des laboratoires publics ou privés. 


\section{LE DROIT FRANÇAIS DES CONCOURS ENTRE PERMANENCE ET ÉVOLUTION}

\section{Delphine Espagno}

École nationale d'administration | « Revue française d'administration publique »

$2012 / 2 n^{\circ} 142$ | pages 369 à 381

ISSN 0152-7401

ISBN 3303337901420

\section{Article disponible en ligne à l'adresse :}

https://www.cairn.info/revue-francaise-d-administrationpublique-2012-2-page-369.htm

Distribution électronique Cairn.info pour École nationale d'administration.

(C) École nationale d'administration. Tous droits réservés pour tous pays.

La reproduction ou représentation de cet article, notamment par photocopie, n'est autorisée que dans les limites des conditions générales d'utilisation du site ou, le cas échéant, des conditions générales de la licence souscrite par votre établissement. Toute autre reproduction ou représentation, en tout ou partie, sous quelque forme et de quelque manière que ce soit, est interdite sauf accord préalable et écrit de l'éditeur, en dehors des cas prévus par la législation en vigueur en France. Il est précisé que son stockage dans une base de données est également interdit. 


\title{
LE DROIT FRANÇAIS DES CONCOURS ENTRE PERMANENCE ET ÉVOLUTION
}

\author{
Delphine ESPAGNO
}

Maître de conférences en droit public, Institut d'études politiques de Toulouse

\section{Résumé}

Les concours administratifs constituent une étape nécessaire au recrutement dans la fonction publique française. Soumis à de nombreux principes juridiques, l'organisation et le déroulement des concours supposent le respect des règles de droit sous l'œil attentif de la juridiction administrative. La modernisation des concours administratifs, liée à l'évolution de la fonction publique et de ses modes de recrutement, entraine une adaptation des épreuves et des règles applicables en la matière. Parfois sévèrement remis en cause, le concours demeure toutefois le meilleur outil de recrutement dans la fonction publique

\section{Mots-clefs}

Concours, fonction publique, droit public, égalité d'accès, réforme

\begin{abstract}
- French law on entrance examinations caught between permanence and change-Civil service entrance examinations are a necessary step for recruitment to the French civil service. Subject to numerous legal principles, the organisation and conduct of the examinations implies adherence to the rules of law, under the watchful eye of the administrative jurisdiction. The modernisation of civil service entrance examinations, linked to the development of the civil service and its recruitment methods, results in an adaptation of the tests and the rules applicable thereto. Although sometimes severely called into question, the entrance examination remains the best tool for recruitment to the civil service.
\end{abstract}

\section{Keywords}

I Entrance examination, civil service, public law, equal access, reform

Les concours administratifs constituent, en France, le mode privilégié de recrutement dans la fonction publique, en tout cas encore pour quelques années. En effet, comme le soulignent certains auteurs, « la pratique administrative française s'est depuis longtemps orientée vers une généralisation du procédé du concours de recrutement. Elle y voit le double avantage d'éliminer les risques d'arbitraire dans le choix des agents 
et d'assurer au maximum les garanties de qualification par les moyens d'une sélection technique ${ }^{1}$. Comme l'écrivait Léon Duguit dans son Traité de droit constitutionnel, «malgré les inconvénients que présente ce système de recrutement, c'est encore celui qui offre le plus de garanties contre l'arbitraire et le favoritisme $»^{2}$. Pourtant les concours administratifs ne sont pas épargnés par la critique. On leur reproche d'être coûteux, d'être un mode de recrutement confinant à la reproduction sociale, d'être fondés sur une logique beaucoup trop scolaire ou encore de ne plus permettre l'accès à la fonction publique aux plus modestes...Tant de critiques auxquelles pourtant ce mode de recrutement résiste... Adapté, remodelé, réformé, il n'en demeure pas moins le dispositif le plus utilisé pour recruter dans la fonction publique en France ${ }^{3}$ aujourd'hui encore.

Depuis $1941^{4}$, le principe du recrutement est fondé dans la fonction publique française sur le concours. Ce principe est d'ailleurs régulièrement réaffirmé par les différents statuts de la fonction publique depuis $1946^{5}$. Mais comme l'a démontré Pierre Sadran ${ }^{6}$, la notion de concours n'est en réalité pas évidente. Par concours administratif, il faut entendre « un procédé de recrutement de la fonction publique tendant à la désignation, par un jury, à la suite d'épreuves appropriées, du ou des candidats aptes à être nommés par l'autorité compétente $»^{7}$. Cette définition n'est pas réellement suffisante pour distinguer le concours de l'examen. Dans le cadre d'un concours, le nombre de places offertes est limité alors que la réussite à un examen n'est conditionnée que par l'obtention d'un résultat prédéterminé dans le règlement d'examen. À la différence de l'examen, le concours constitue un procédé de sélection et non une opération de vérification d'une aptitude. La jurisprudence du Conseil d'État a eu, à plusieurs reprises, à se prononcer sur l'identification d'un concours ${ }^{8}$. L'élément essentiel dans le concours réside dans le caractère déterminant que jouent les épreuves dans le choix des agents, laissant une marge de manœuvre réduite aux autorités compétentes pour nommer. À cela s'ajoute le fait que le concours suppose que les qualités des candidats soient appréciées par un jury indépendant de l'autorité hiérarchique, que le nombre de places proposé soit limité et qu'il existe soit un classement des candidats par ordre de mérite, soit une simple liste d'aptitude présentée par ordre alphabétique (sans hiérarchie des mérites respectifs des canditats).

En application de cette définition et de ces précisions, on peut considérer qu'il existe en droit de la fonction publique française une diversité de concours : concours sur

1. Laubadere (André de), Gaudemet (Yves), Traité de droit administratif, Tome 5, 2000, Paris, LGDJ, ${ }^{\circ} 125$.

2. Duguit (Léon), Traité de droit constitutionnel, tome 3, 2e édition, Paris, Boccard p. 41.

3. À titre anecdotique, plus de 400 concours administratifs sont organisés chaque année en France selon la DGAFP mais plus d'un millier selon le Rapport de la mission préparatoire au réexamen général du contenu des concours d'accès à la fonction publique d'Etat établi par Corinne DESFORGES et J.G de Chalvron, Janvier 2008 , p. 9.

4. L'article 27 de la loi du 14 septembre 1941 dispose que « nul ne peut être admis à un emploi de début s'il n'a satisfait aux épreuves d'un concours ou aux examens de sortie d'une école lorsque le recrutement est assuré par cette voie».

5. Article 27 et 28 de la loi du 19 octobre 1946 reconnu comme étant le premier véritable statut général de la fonction publique, article 18 de l'ordonnance de 1959 et enfin l'article 16 de la loi de 1983 : «Les fonctionnaires sont recrutés par concours sauf dérogation prévu par la loi ». p. 1 à 49 .

6. Sadran (Pierre), Le régime juridique des concours de la fonction publique, Thèse Bordeaux I, 1972,

7. Cornu (Gérard), Vocabulaire juridique, l'Association Henri Capitant, Quadrige, PUF, 2000.

8. Voir en ce sens, CE 6 juin 1962, Dioudonnat, Rec., p. 990. CE Ass., 13 juillet 1967, Geslin, Rec., p. 316. Ainsi dans cette espèce, le Conseil d'État précise que «Considérant que le nombre des emplois d'externe des hôpitaux offerts aux candidats est, chaque année, limité ; qu'il est pourvu à ces emplois d'après un classement qui résulte de notes obtenues par les intéressés ; qu'ainsi les opérations qui aboutissent à la nomination des externes des hôpitaux ont le caractère d'un concours ». 
épreuves et concours sur titre, concours externe, interne et troisième concours, concours spécialisés et concours généralistes.

Inscrits dans un cadre juridique relativement précis, les concours administratifs sont à la fois l'objet de dispositions législatives et réglementaires, telles que le Statut général de la fonction publique, mais sont parfois soumis au regard du juge administratif qui dégage les principes généraux applicables en la matière. Encadrés par les textes, appréciés par les magistrats de l'ordre administratif, les concours sont soumis aux évolutions de la fonction publique et de la réforme de l'État.

Depuis quelques années, nous le savons, le droit de la fonction publique connaît des évolutions notables liées principalement à une volonté d'amélioration budgétaire ${ }^{9}$ mais aussi à une adaptation au droit communautaire et aux enjeux démographiques ${ }^{10}$. Objet privilégié de la Révision générale des politiques publiques, la fonction publique est soumise à de nombreuses évolutions et modifications. Dans ce contexte, le droit des concours n'est pas épargné par le vent de la réforme. L'organisation des concours, le contenu des concours de la fonction publique ont tour à tour retenu l'attention des pouvoirs publics. Le Président de la République lui-même a considéré qu'il était nécessaire de redéfinir le contenu des épreuves des concours administratifs dénonçant leur inadaptation au métier de fonctionnaire. Dans un discours prononcé à Nantes, il a exposé son souhait de refonder la fonction publique pour une meilleure réforme de l'État. Principalement, le Président de la République a précisé qu'il fallait rénover les modes de recrutement et les diversifier. Selon le chef de l'État, « si le concours continue d'être la seule et unique règle pour la promotion » et « si les épreuves restent aussi académiques, aussi peu en rapport avec les compétences et l'expérience de chacun », il ne sera pas possible de moderniser la fonction publique ${ }^{11}$.

Malgré cette volonté de réformer la fonction publique en commençant par les modes de recrutement et en poursuivant par la gestion des carrières des agents, les concours administratifs sont toujours une étape nécessaire organisée dans le respect des règles et des procédures dont le déroulement et l'issue sont également soumis à un certain formalisme juridique. Enfin, les concours aujourd'hui sont soumis, en France, à des évolutions faisant émerger une tendance à la professionnalisation et à la prise en compte de situations professionnelles et d'expériences professionnelles. Ces transformations peuvent conduire à s'interroger sur la pertinence des évolutions des conditions de recrutement dans la fonction publique aujourd'hui et demain.

\section{LE CONCOURS : PROCÉDÉ DE RECRUTEMENT DE DROIT COMMUN STRICTEMENT ENCADRÉ}

Le concours administratif est le procédé de recrutement de droit commun, même si on peut avoir recours dans la fonction publique à d'autres modes de recrutement, comme

9. Chevalier (Yves), « La réforme budgétaire et la gestion des ressources humaines : quelles conséquences pour la fonction publique ? », AJDA, 2006, p. 523 et suivantes.

10. Voir en ce sens, le Rapport annuel du Conseil d'État, Perspectives pour la fonction publique, La documentation française, Paris, 2003 ainsi que Silicani (Jean Ludovic), Livre blanc sur l'avenir de la fonction publique : faire des services publics et de la fonction publique des atouts pour la France, La documentation française, Paris, 2008.

11. Discours prononcé le 19 septembre 2007 à l'IRA de Nantes, http://www.elysee.fr/president/ les-actualites/discours/2007/institut-regional-d-administration-ira.8293.html. 
le contrat par exemple ${ }^{12}$. Il présente l'avantage d'assurer un caractère relativement démocratique du recrutement et de permettre le recrutement de candidats de qualité.

\section{Le principe de l'égal accès à l'emploi public}

Le système du recrutement par concours permet de mettre en œuvre un principe fondamental de notre droit posé dans la Déclaration des droits de l'homme et du citoyen selon lequel tous les citoyens doivent avoir un égal accès à l'emploi public ${ }^{13}$. Ce principe constitue un principe fondamental applicable de manière systématique en matière de recrutement dans la fonction publique. Il est le principe commun, nécessaire et préalable à l'organisation de tous les concours administratifs.

Non directement exprimé par le Statut avant la loi du 13 juillet 1983, le principe de l'égale admissibilité aux emplois publics constitue un principe général du droit public en application de la jurisprudence classique Barel de $1954^{14}$. Le Conseil d'État a souvent été amené à consacrer cette règle et le Conseil Constitutionnel à en faire l'interprétation dans plusieurs décisions ${ }^{15}$. La particularité de ce principe fondamental en matière de concours de la fonction publique est qu'il est affirmé dans l'article 1er de la Constitution, l'article 6 de la Déclaration des droits de l'homme et du citoyen et le préambule de la Constitution de 1946. Le Conseil d'État fait une référence régulière, depuis 1988, à la source constitutionnelle du principe ${ }^{16}$ influencé par l'abondante jurisprudence du Conseil constitutionnel en la matière ${ }^{17}$.

Ce principe ne souffre que de peu d'exceptions en droit de la fonction publique. Ainsi, à partir de son application se dégage une série de conséquences importantes conduisant à l'impossibilité d'écarter un candidat à une fonction publique en raison de ses opinions religieuses ${ }^{18}$, l'interdiction des discriminations fondées sur les opinions politiques qu'il s'agisse de favoriser un candidat en raison de ses opinions politiques ou au contraire de le pénaliser. Enfin, en application de ce principe fondateur pour le droit des concours, les discriminations fondées sur le genre sont interdites. La reconnaissance de cette interdiction s'est faite en plusieurs étapes en matière de concours, alliant le législateur et le juge administratif. On reconnaît donc l'existence d'une égalité des genres pour l'accès à la fonction

12. Quelques chiffres : le recrutement par concours externe (externe, troisième voie, concours unique) est de 97,6 \% selon le Rapport annuel sur l'état de la fonction publique, Faits et chiffres, 2008-2009, volume 1, DGAFP, La documentation française, Paris 2009, p.433. La répartition se fait de la manière suivante : $91,4 \%$ par concours externe, $2,1 \%$ par la troisième voie, $4,1 \%$ par concours unique. Parmi ces recrutements $70,3 \%$ sont des catégories A, $17 \%$ des catégories B et $12 \%$ des catégories C en sachant que pour chacune de ces catégories, notamment A et B, les candidats sont en général surdiplômés.

13. Article 6 de la Déclaration des droits de l'homme et du citoyen : «tous les citoyens étant égaux à ses yeux sont également admissibles à toutes les dignités, places et emplois publics, selon leur capacité, et autre distinction que celle de leurs vertus et de leurs talents ».

14. CE 28 mai 1954, Barel, Rec. p. 308.

15. Conseil constitutionnel, 14 janvier 1983, déc. $\mathrm{n}^{\circ} 82-153$ DC, loi relative au statut général des fonctionnaires, Rec. p.35 ; Conseil Constitutionnel 30 août 1984, déc. № 84-178 DC, loi portant statut du territoire de la Nouvelle-Calédonie et dépendances et notamment ses articles 12, 131 et 137, Rec. p. 69.

16. CE Ass 16 décembre 1988, Bleton, Rec. p. 451.

17. Voir en ce sens, Conseil constitutionnel 24 avril 2003, Loi relative aux assistants d'éducation.

18. La jurisprudence du Conseil d'État en la matière est abondante. CE 10 mai 1912, Abbé Bouteyre, $R e c .$, p. 553 à propos de la légalité de la mesure d'un ministre qui refuse à un abbé de se présenter à l'agrégation de philosophie. Il faut remarquer qu'aujourd'hui la question ne serait sans doute pas traitée de la même manière, le Conseil d'État ayant accepté l'entrée dans l'enseignement public d'un professeur qui au cours d'une période de disponibilité avait embrassé l'état ecclésiastique. Voir en ce sens CE AG 21 septembre 1972, Les grands avis du Conseil d'État, 3 édition, 2008, p. 100. 
publique ${ }^{19}$. Après différentes étapes jurisprudentielles et législatives, la loi du 9 mai $2001^{20}$ vient modifier le Statut général de la fonction publique en ajoutant un article 6 bis précisant « qu'aucune distinction ne peut être faite entre les fonctionnaires en raison de leur sexe ». Cependant, cette interdiction ne signifie pas l'impossibilité d'instaurer des dérogations ${ }^{21}$ au principe quand «des recrutements distincts pour les femmes ou les hommes peuvent, exceptionnellement, être prévus lorsque l'appartenance à l'un ou à l'autre sexe constitue une condition déterminante de l'exercice des fonctions ${ }^{22}$. À propos de l'accès à la fonction publique, la loi de 2001 innove. En effet, elle prévoit la possibilité de distinguer entre les femmes et les hommes en vue de la désignation par l'administration des membres des jurys et différents comités de sélection. En la matière, le principe de l'égalité des sexes est parfois difficilement appliqué. C'est sans doute dans l'organisation des concours de l'enseignement supérieur que l'instauration de ces dispositions a posé le plus de difficultés. Dans le cadre de l'organisation des concours de l'enseignement supérieur, par exemple, l'introduction de quotas de femmes dans les jurys, en application de la loi de 2001 et du décret du 3 mai $2002^{23}$, a donné lieu à un certain nombre de controverses ${ }^{24}$.

Or si ce principe juridique représente un élément essentiel en matière de recrutement dans la fonction publique, il s'applique tout au long de la carrière des agents de la fonction publique ${ }^{25}$. Malgré l'instauration de ces quotas, et une volonté d'opérer un renforcement juridique de la parité en matière professionnelle, les femmes, bien que largement majoritaires dans la fonction publique, n'occupent des fonctions d'encadrement et de responsabilité qu'à une hauteur relativement limitée ${ }^{26}$.

Outre ces principes généraux qui constituent les corollaires du principe fondamental d'égalité d'accès à l'emploi public, le recrutement dans la fonction publique peut être soumis à des conditions particulières : possession de la nationalité française, jouissance des droits civiques, aptitude physique, limite d'âge. Ces conditions particulières évoluent notamment au regard de la construction communautaire et des contraintes juridiques nées de la signature des traités communautaires. Ainsi on sait que l'exigence

19. La jurisprudence administrative sur le sujet est également abondante et importante. Pendant longtemps, le juge administratif a admis qu'il était possible pour des raisons tirées de l'intérêt du service d'exclure les femmes à l'accès à certaines fonctions (CE 3 juillet 1936, Dlle Bobard, Rec., p. 721).

20. Loi n²001-397 du 9 mai 2001 relative à l'égalité professionnelle entre les femmes et les hommes, JORF, $\mathrm{n}^{\circ} 108,10$ mai 2001, p. 7320.

21. Aujourd'hui, la liste de ces dérogations est relativement brève. Elle comporte notamment les surveillants extérieurs de l'administration pénitentiaire : CE 11 mai 1998, Melle Aldige, RFDA 1998, p. 890, Ces dérogations sont maintenues malgré le fait que la CJCE ait estimé que la France manquait aux obligations lui incombant en vertu du Traité de Rome (CJCE 30 juin 1988, RFDA 1988, p.978).

22. Article 6 bis de la loi ${ }^{\circ} 83-634$ du 13 juillet 1983 portant droits et obligations des fonctionnaires.

23. Décret $n^{\circ} 2002-766$ du 3 mai 2002 relatif aux modalités de désignation, par l'administration, dans la fonction publique de l'État, des membres des jurys et des comités de sélection et de ses représentants au sein des organismes consultatifs.

24. Lichere (F.) et Viala (V), « La légalité des quotas de sexe (pour certains jurys de concours) », AJDA, 2003 , p. 817 et suivantes. Cassia (Paul), «La légalité des quotas par sexe sauf pour les jurys des concours d'agrégation de l'enseignement supérieur », AJDA, 2003, p. 825.

25. Ainsi, le Conseil d'État n'admet pas la constitution de conseils de discipline de composition différente pour sanctionner des hommes ou des femmes (CE 26 juin 1989, Fédération éducation nationale, RFDA, 1985, p.714) comme il sanctionne également les discriminations « à rebours » en faveur des femmes (CE 5 décembre 1984, Synd. Ind., RFDA, 1985, p. 548).

26. DGAFP, Rapport annuel sur l'état de la fonction publique, Faits et chiffres 2009-2010, volume 1, La documentation française, Paris, 2010, p. 271 et suivantes. Les femmes représentent 44,3\% de 1'effectif total dans la fonction publique d'État hors enseignement (20,3\% emplois de direction), $61 \%$ effectif total dans la fonction publique territoriale (18\% fonctions supérieures d'encadrement). Elles représentent 76,7 \% de l'effectif global de la fonction publique hospitalière avec une représentation à hauteur de 40,2\% dans les fonctions supérieures d'encadrement. 
de nationalité n'est plus opposable aux ressortissants étrangers membres de l'Union européenne pour l'accès au corps, cadre d'emplois et emplois dont les attributions « soit ne sont pas séparables de l'exercice de la souveraineté, soit ne comportent aucune participation directe ou indirecte à l'exercice de prérogatives de puissance de l'État ou des autres collectivités publiques $\gg{ }^{27}$. Cette ouverture de la fonction publique à des non-nationaux constitue une innovation juridique importante en matière de recrutement ces dernières années. Toutefois, si elle est importante d'un point de vue juridique, elle ne modifie pas considérablement le visage du recrutement, notamment par concours. En effet, les candidats étrangers doivent remplir les mêmes conditions que les candidats français.

\section{Les opérations complexes nécessaires à l'organisation des concours}

Au-delà de ces principes applicables à tous les concours, chaque concours fait l'objet d'une réglementation abondante et d'une jurisprudence apportant des précisions essentielles par l'édiction de «principes généraux applicables même sans texte, en matière d'organisation de concours ». Quel que soit le concours à organiser, il s'agit d'une opération complexe d'un point de vue juridique supposant l'adoption de plusieurs types d'actes administratifs, dont chacun est susceptible de donner lieu à un contentieux. Ainsi, le concours ne débute que par une série d'opérations préalables au déroulement du concours : l'ouverture du concours et la vérification des conditions susceptibles de donner lieu à une admission à concourir. Ces préalables administratifs sont obligatoires. Ils entraînent pour l'administration organisatrice du concours un temps nécessaire à la mise en œuvre de ces procédures, et par conséquent un coût. Ces opérations alourdissent la procédure de recrutement pour l'administration, mais ont pour objectif de veiller au respect des principes précédemment exposés et d'assurer aux candidats une égalité de traitement dans le déroulement du concours. Tous les candidats doivent être placés dans une situation identique pour pouvoir concourir dans les meilleures conditions. Ainsi, la différence entre les candidats est supposée ne se faire que sur les qualités propres de chacun d'entre eux, sans influence du contexte et du déroulement du concours. On met donc en place une égalité « fictive » des candidats pour assurer à chacun une chance de réussir.

L'organisation du concours doit être faite par l'autorité compétente à une fréquence en principe annuelle, sauf s'il existe une disposition contraire prévoyant une réduction de la fréquence ou son augmentation au regard des besoins. L'administration n'est pas tenue, en principe, d'ouvrir un concours en cas de vacances d'emplois dans un $\operatorname{corps}^{28}$. En la matière, elle dispose d'un pouvoir discrétionnaire qui lui octroie également la possibilité de retirer sa décision d'ouverture d'un concours, même si des candidats ont déjà déposé leur dossier d'inscription. En droit administratif, la décision d'ouverture d'un concours ne constitue pas une décision créatrice de droits. En revanche, l'ouverture d'un concours est soumise à une procédure de publicité qui l'officialise permettant ainsi aux candidats de prendre connaissance de la tenue de ce dernier. La publication - au Journal officiel si le concours est national - de l'avis d'ouverture du concours est un acte insusceptible de recours pour excès de pouvoir. La légalité de cette procédure peut uniquement être

27. Loi $n^{\circ} 91-715$ du 26 juillet 1991 portant diverses mesures relatives à la fonction publique introduit un article 5 bis dans la loi du 13 juillet 1983 .

28. CE, 31 mars 1993, Vaillant, Rec., p. 810. 
contestée par voie d'exception au moment de la contestation des résultats du concours. Dans ce cas, s'il s'avère que les conditions de légalité de la publicité n'ont pas été remplies, le concours peut être annulé. L'avis d'ouverture doit contenir toutes les informations nécessaires pour s'inscrire au concours. Une fois la date de clôture des inscriptions dépassée, la réglementation du concours ne peut plus être modifiée. En revanche, le nombre de places peut, quant à lui, être modifié en toute légalité jusqu'au commencement des épreuves ${ }^{29}$. Le jury, nommé par l'autorité organisatrice du concours, est toutefois indépendant. La composition du jury est fixée par la réglementation du concours.

Une fois le concours ouvert, l'administration doit examiner les dossiers des candidats et vérifier qu'ils remplissent les conditions requises pour être admis à concourir. Le Statut général de la fonction publique, dans ses articles 5 et 5 bis, énonce les conditions générales applicables à l'ensemble des concours. La réglementation spécifique peut poser des conditions supplémentaires propres au concours organisé. Ainsi, l'administration va établir une liste des candidats admis à concourir ce qui lui laisse la possibilité d'écarter des candidats au motif qu'ils lui paraissent ne pas satisfaire aux conditions exigées ou parce qu'ils ne correspondent pas à l'intérêt du service. Sur cet aspect de l'admission à concourir, l'administration dispose d'un pouvoir discrétionnaire qui lui donne la possibilité d'écarter purement et simplement des candidats qu'elle juge indésirables. Mais tout en laissant cette possibilité à l'administration, le juge administratif veille sévèrement à ce que l'exclusion de la candidature a bien eu lieu réellement dans l'intérêt du service et s'est faite dans le respect du principe d'égale admissibilité aux emplois publics ${ }^{30}$. Les candidats se doivent de remplir les conditions posées par le statut au moment de la première épreuve du concours ou de la première réunion du jury s'il s'agit d'un concours sur titre.

En pratique, le suivi de ces procédures alourdit considérablement l'organisation des concours. Ainsi, l'administration n'a parfois pas le temps de faire toutes les vérifications nécessaires avant le début des épreuves. En effet, le nombre des candidats inscrits au concours administratif, donc de dossiers déposés, est souvent élevé. Ces difficultés matérielles sont telles que le législateur autorise l'administration à réaliser une vérification des conditions à la fin du concours, voire au moment de la nomination des lauréats ${ }^{31}$.

L'ensemble de ces règles révèle bien « une grande complexité dans l'organisation des concours ${ }^{32}$ laissant aux autorités organisatrices une marge de manœuvre importante sur certains points et, au contraire, les contraignants par des aspects très formels. Ces opérations juridiques complexes pourraient en effet entamer la malléabilité du concours au bénéfice d'un choix de l'organisation d'un recrutement par la voie contractuelle.

Une fois ces opérations préliminaires terminées, le concours est alors ouvert. Il doit en principe se dérouler conformément aux règles applicables à cette phase du recrutement.

29. CE, 6 mai 1996, Pilorge, Req. $\mathrm{n}^{\circ} 158181$.

30. Ont été jugés légaux les refus d'admission à concourir suivants : - celui d'un candidat à l'ENM ayant participé antérieurement à la diffusion d'un journal d'opinion incompatible avec la réserve nécessaire s'imposant à un futur magistrat (CE 10 juin 1983, Raoult, Req. $\mathrm{N}^{\circ} 34832$; celui d'une candidate aux fonctions d'agent de surveillance de Paris ayant commis antérieurement un vol à l'étalage (CE 25 octobre 2004, Préfet de police, Req. $\left.\mathrm{N}^{\circ} 256944\right)$.

31. Loi n ${ }^{\circ} 84-16$ du 11 janvier 1984 portant dispositions statutaires relatives à la fonction publique de l'État, article 20 : «S'il apparât, au moment de la vérification des conditions requises pour concourir, laquelle doit intervenir au plus tard à la date de la nomination, qu'un ou plusieurs candidats déclarés aptes par le jury ne réunissaient pas lesdites conditions, il peut être fait appel, le cas échéant, aux candidats figurant sur la liste complémentaire ».

32. Rapport de la mission préparatoire au réexamen général du contenu des concours d'accès à la fonction publique d'État, précité, p. 10. 


\section{LE DÉROULEMENT DES CONCOURS : COLLÉGIALITÉ, SOUVERAINETÉ ET IMPARTIALITÉ}

Le déroulement des épreuves est assuré par un jury, organe collégial et souverain présentant des garanties d'impartialités.

\section{La collégialité, unicité et souveraineté du jury constituent des garanties d'équité}

Ce jury est indépendant de l'autorité organisant le concours. Une fois nommé, il n'est plus lié à elle, pas plus, en principe, qu'à aucune autre autorité administrative ou politique. En d'autres termes, aucune autorité n'est susceptible de pouvoir exercer des pressions sur les membres du jury, ni l'autorité administrative, ni politique. Cette indépendance du jury est assortie d'une autre qualité, il est en principe souverain. La souveraineté du jury signifie que, dans le respect des règles du concours, l'appréciation portée par le jury sur les qualités des candidats est juridiquement incontestable et qu'elle s'impose à tous, candidats comme administration. Elle entraîne, en principe, l'irrecevabilité d'un recours pour excès de pouvoir contre une décision collégiale du jury ${ }^{33}$. En revanche, elle ne donne pas la possibilité aux membres des jurys de déroger aux règles préalablement fixées. Ainsi, un jury ne peut interroger un candidat sur un thème ne faisant pas l'objet du programme. Lors du déroulement d'un concours de recrutement de professeurs des universités, le jury ne peut soumettre à un candidat un document en langue étrangère ${ }^{34}$, ni ajouter une épreuve complémentaire ${ }^{35}$. Il est tenu de respecter les critères d'évaluation prédéterminés et la cotation des épreuves. Il n'est pas libre de fixer la note éliminatoire ${ }^{36}$. En dehors des réserves émises, le jury n'a pas à motiver ses délibérations et dispose d'un pouvoir discrétionnaire de décision grâce auquel il bénéficie d'une large marge de manœuvre. Le juge administratif refuse de contrôler si le jury a correctement apprécié la valeur des titres du candidat ou des épreuves qu'il a subies ${ }^{37}$.

En matière de concours administratif, l'appréciation du jury doit s'exercer de manière discrétionnaire mais non arbitraire. Cette liberté d'appréciation s'exerce dans le respect des principes juridiques qui s'ajoutent au règlement du concours. L'objectif est à la fois de laisser aux membres du jury une latitude suffisante pour apprécier la valeur du candidat et d'aménager suffisamment de garanties au profit de ce dernier. La collégialité du jury constitue une assurance supplémentaire de justesse, d'honnêteté et de compétence. Elle prend tout son sens au moment de la délibération du jury. Cette collégialité a pour corollaire l'unicité du jury qui est une manifestation supplémentaire de l'égalité de traitement des candidats. Unicité et collégialité n'interdisent pas que, pour des raisons matérielles liées au nombre de candidats, le jury se subdivise en plusieurs groupes d'examinateurs ${ }^{38}$, à condition qu'il opère ensuite une péréquation des notes attribuées par chaque groupe d'examinateurs ${ }^{39}$.

33. CE, 12 décembre 1984, Foresti, RFDA 1985, p. 731 et CE 20 mars 1987, Gambus, Dalloz 1987, IR, p. 83. Dans ces affaires, le CE a considéré que l'appréciation de la valeur des épreuves relève uniquement du jury et qu'il ne revient pas au juge administratif d'en faire le contrôle.

34. CE, 2 mai 1990, Barbier, RDP, 1991, p. 876.

35. CE, 19 mai 1993, Geneix, RDP, 1994, p. 1858.

36. CE, 26 avril 2000, Amouri, Req. ${ }^{\circ} 190423$.

37. CE, 22 février 1995, Bonneville, Req. $\mathrm{n}^{\circ} 151130$.

38. CE, 11 juin 2004, Maurin et Masson, Req. $\mathrm{N}^{\circ} 253787$.

39. Aguila (Yann), « Subdivision d'un jury de concours et principe d'égalité des candidats », AJDA, 2005 , p. 204. 


\section{L'impartialité du jury est une condition essentielle au bon déroulement du concours}

La partialité du jury est mise en cause devant le juge administratif ${ }^{40}$. Elle résulte de l'existence de liens particuliers entre un des membres du jury et un candidat, liens professionnels ou personnels. Un candidat peut se retrouver ainsi lors d'un concours face à son supérieur hiérarchique, membre du jury. Dans ce cas, le manque d'impartialité d'un des membres du jury est reconnu. Le juge administratif opère un contrôle relativement sévère du manque de respect de l'impartialité. Dans une décision de juillet 2008, le Conseil d'État a été amené à préciser les contours de cette notion d'impartialité des membres d'un jury d'examen. Une requérante contestait le fait que le jury avait écarté l'un de ses membres au motif qu'il la connaissait. Dans ce genre de situation, il est en effet d'usage que le membre du jury n'interroge pas un candidat qu'il connaît, ne le note pas et ne participe pas la discussion le concernant lors des délibérations. La Haute juridiction administrative a donné raison à la requérante qui remettait en cause cette pratique. À l'occasion de cette affaire, le Conseil d'État a précisé que « la seule circonstance qu'un membre d'un tel jury d'examen professionnel connaisse un candidat ne suffit pas à justifier qu'il s'abstienne de participer aux délibérations qui concernent ce candidat ${ }^{41}$. À cette considération, le juge ajoute quelques nuances en considérant toutefois que le respect du principe d'impartialité s'oppose à ce qu'un membre du jury participe aux interrogations et aux délibérations d'un candidat avec lequel il a des relations personnelles ou professionnelles, liens qui selon le Conseil d'État seraient susceptibles d'influer sur son appréciation. La question de l'impartialité des jurys d'examens professionnels comme des concours constitue un point sur lequel le juge administratif fait preuve d'un degré d'exigence important. Mais pour autant, le rapporteur public, dans les conclusions qu'il rend dans cette affaire, ne manque pas de considérer qu'il convient de laisser le soin aux jurys d'apprécier cette question de l'impartialité sans pour autant « céder à cette tyrannie de l'apparence ${ }^{42}$. Le respect de la garantie d'impartialité légitime non seulement le concours lui-même et l'organisme qui le gère, mais aussi l'autorité des membres du jury. Enfin, le respect de l'impartialité assure aux candidats une égalité de traitement au moment du déroulement des épreuves. Or, on sait combien les juridictions administratives sont sourcilleuses du respect de cette égalité. Le lien entre impartialité et égalité est ténu. En effet, le fait qu'un membre du jury s'abstienne de prendre part à l'interrogation sans pour autant quitter le jury ne remet pas en cause l'égalité des candidats. Par conséquent, l'impartialité dite subjective n'implique nullement qu'un membre du jury se retire parce qu'il a connaissance d'un candidat ${ }^{43}$. En revanche, lorsque se pose un problème d'impartialité objective, que le jury est confronté à une proximité entre l'un de ses membres et un candidat, alors l'impartialité induit un problème d'égalité. Le fait d'avoir une relation professionnelle ou des relations personnelles avec l'un des candidats entraîne une impartialité objective de l'un des membres du jury et remet en cause l'égalité, principe essentiel en matière de droit de concours.

Le président du jury est le garant de cette égalité et du respect de l'impartialité des membres du jury. Lorsqu'arrive la fin des épreuves et après avoir délibéré collégialement, le jury établit une liste des candidats admis, par ordre de mérite dans les fonctions publiques d'État et hospitalière. Dans la fonction publique territoriale, le jury établit une

40. Aguila (Yann), «L'impartialité du jury d'un examen professionnel », AJDA, 2008, p. 2124.

41. CE Section 18 juillet 2008, Madame B., Req. $N^{\circ} 291957$.

42. Aguila (Yann), op.cit., p. 2125.

43. Voir en ce sens, CE 22 juin 2011, Roche Gambini, Req n 336757. 
liste d'aptitude par ordre alphabétique pour la liste principale conformément aux dispositions de l'article 15 du décret du 20 novembre $1985^{44}$. En application de dispositions réglementaires, il est possible d'établir une liste complémentaire afin de faire face à d'éventuels désistements ou à des vacances imprévues dans les corps concernés par le concours organisé. Le décret du 18 juin 2003 reconnaît la possibilité d'établir, pour tous les concours de recrutement dans la fonction publique d'Etat, une liste complémentaire par ordre de mérite ${ }^{45}$. Il faut toutefois savoir que le jury n'est pas tenu d'attribuer toutes les places mises au concours en fonction du niveau des candidats, il peut estimer ne pas pourvoir tous les postes ouverts.

Une fois la liste des admis publiée, le concours est terminé. Dans la majorité des cas, le concours se termine bien pour l'autorité administrative organisatrice. La publication des résultats n'entraîne pas pour l'administration une obligation de nomination. Elle n'est pas tenue juridiquement de nommer les candidats proposés par le jury : elle peut n'en nommer aucun, elle peut n'en nommer que certains dans le respect de l'inscription sur la liste principale. Dans la fonction publique territoriale, les collectivités territoriales ont une plus grande marge de manœuvre. Le fait d'être lauréat d'un concours de la fonction publique territoriale n'entraîne pas de manière systématique le recrutement. Les candidats admis sur la liste d'aptitude doivent procéder eux-mêmes à la recherche d'un emploi. Dans les autres fonctions publiques, l'occupation d'un emploi à l'issue du concours est quasiment assurée. L'obtention du concours conduit soit à la nomination dans un corps ou dans un cadre d'emploi, soit à l'affectation dans une école de formation.

Plus rarement, l'issue du concours peut déboucher sur une période contentieuse au cours de laquelle un candidat évincé, un membre d'un corps ou d'un cadre d'emplois concerné par le concours, un syndicat de fonctionnaires cherchent à obtenir juridiquement l'annulation des opérations du concours. La contestation contentieuse se fait sur l'ensemble du concours et non sur la nomination intuitu personae. Les juridictions administratives peuvent décider - Conseil d'État pour les concours nationaux, tribunaux administratifs pour les concours locaux -, si elles estiment qu'il y a un doute sérieux quant à la légalité du déroulement du concours, ou de l'une des opérations complexes nécessaires à l'ouverture du concours, de limiter son annulation au cas individuel ou à tout le concours. Cette situation s'est produite, par exemple, à propos du classement de sortie de l'ENA ${ }^{46}$ et également à propos des délibérations d'une commission de spécialistes recrutant des professeurs d'université ${ }^{47}$.

Le droit des concours consiste en la mise en œuvre de principes, de règles, qui apparaissent comme particulièrement rigides. Le juge administratif, garant de la légalité, vérifie lorsque cela est nécessaire, le respect de l'ensemble de ces principes juridiques.

Depuis quelques années, dans le cadre d'une modernisation de la fonction publique, les pouvoirs publics mettent en avant la nécessité de faire évoluer les procédés de recrutement et donc les concours. Ces évolutions, qui se caractérisent par une nette tendance au développement d'épreuves de professionnalisation au concours externe comme au concours interne, posent un certain nombre de difficultés pratiques comme juridiques.

44. Décret $\mathrm{n}^{\circ}$ 85-1229 du 20 novembre 1985 relatif aux conditions générales de recrutement des agents de la fonction publique territoriale.

45. Décret $\mathrm{n}^{\circ}$ 2003-532 du 18 juin 2003 relatif à l'établissement et à l'utilisation des listes complémentaires d'admission aux concours d'accès aux corps de la fonction publique de l'État, article 1e: « Pour chaque concours organisé en application de l'article 19 de la loi du 11 janvier 1984 susvisée, le jury établit par ordre de mérite une liste complémentaire sur laquelle il fait figurer tous les candidats qu'il estime aptes à exercer les emplois à pourvoir ».

46. CE 10 janvier 2007, Grass, RFDA, 2007, p. 432, Req. n 297864.

47. CE sect. 10 octobre 1997, Lugan, RFDA, 1998, p. 37. 


\section{LA PERTINENCE DES ÉVOLUTIONS DES CONDITIONS DE RECRUTEMENT DANS LA FONCTION PUBLIQUE}

Depuis l'adoption de la circulaire Rocard en 1989, la fonction publique est soumise à de nouveaux objectifs ${ }^{48}$. L'adoption de ce texte marque en matière de service public un véritable tournant. L'objectif est ici de moderniser le service public en assurant une responsabilisation des agents, de développer la formation professionnelle et d'améliorer la qualité du recrutement. Cette étape importante est le point de départ de nouvelles interrogations en matière de fonction publique et d'emploi public. À l'issue de cette période, et notamment à partir du milieu des années 1990, plusieurs rapports ont été consacrés à la fonction publique : le rapport Picq en 1995, le rapport Pochard en $2003^{49}$. Dans celui-ci, la question du recrutement des agents par le biais du concours fait l'objet d'un certain nombre de développements. Il est proposé de diversifier les modes de recrutement pour faire face aux difficultés démographiques afin d'adapter au mieux les qualités des agents aux besoins des services. Marcel Pochard propose de renforcer la diversification des modes d'accès à la fonction publique, notamment à la haute fonction publique, tout en renforçant la professionnalisation.

\section{La modernisation des concours administratifs, une nécessité pour améliorer le recrutement}

Dans le Livre blanc sur l'avenir de la fonction publique, en 2008, Jean-Ludovic Silicani dresse un bilan relativement négatif du recrutement par concours. Ainsi, il relève que les concours sont des opérations lourdes à organiser notamment en raison de la nécessité du respect de nombreux principes juridiques. L'administration est soucieuse de veiller à une sécurisation juridique efficace de ces opérations de recrutement oubliant de se concentrer sur les critères de sélection des candidats. Or, ces concours mettent l'accent essentiellement sur les qualités académiques. Dans le prolongement des propositions faites dans le rapport Pochard, Jean-Ludovic Silicani propose de moderniser l'organisation générale des recrutements et de professionnaliser les modes de recrutement pour le concours externe, notamment. Dans cette perspective, il n'est pas curieux de constater qu'il soit prôné en matière de recrutement un développement plus important du recrutement contractuel, ce qui supprime la lourdeur et la rigidité du concours et permet également le recrutement de profils adaptés aux postes. Autrement dit, dans la logique de la gestion prévisionnelle des emplois, il n'est pas étonnant de faire du concours un obstacle à la modernisation du recrutement. Déjà Marcel Pochard, en 2003, avait insisté sur la nécessité de diversifier les modes de recrutement en accordant une place plus importante au contrat. Les analyses de Jean-Ludovic Silicani sont plus critiques sur le rôle joué par les concours, non qu'il remette en cause le principe même du recours à ce mode de recrutement, mais les concours, tels qu'ils sont organisés dans la fonction publique ne répondent plus aux nécessités du service public. Ainsi, le rapporteur précise qu'il existe de nos jours en France, une " insuffisante adéquation entre la nature des épreuves et les besoins de recrutement des administrations, l'absence de définition des compétences

48. Circulaire du 23 février 1989 relative au renouveau du service public, JORF, 24 février 1989.

49. Rapport annuel du Conseil d'État, Perspectives pour la fonction publique, 2003, La documentation française. 
recherchées et des « viviers » correspondants, enfin, l'absence de formation et d'orientations générales données par l'administration aux jurys $»^{50}$.

Rejoignant le Rapport Desforges et les préconisations du Rapport Dorne-Corraze, J-L. Silicani propose, - et ces propositions sont aujourd' hui effectives dans certains concours de la fonction publique (par exemple au niveau territorial) - « d'organiser des concours comprenant trois composantes : la première serait de nature professionnelle (étude de cas ou mise en situation); la deuxième tendrait à apprécier le niveau général des candidats [ ] ; la troisième serait centrée sur son potentiel humain ; le cas échéant, des épreuves spécifiques, orales ou écrites, pourraient être organisées pour chaque spécialité d'un cadre statutaire ou d'un cadre d'emplois ${ }^{51}$. Aux fins de modernisation, l'accent est mis sur la dimension professionnalisante des épreuves des concours d'accès à la fonction publique. Le développement d'épreuves du type de la reconnaissance des acquis de l'expérience professionnelle, les épreuves orales d'entretien avec les jurys qui ont pour objectif de placer les candidats en situation professionnelle modifient progressivement le visage des concours. La disparition dans certains concours des épreuves de culture générale sous forme de dissertation, ou encore de dissertation de droit public au profit d'épreuves sous forme de questions appelant une réponse courte est significative de l'évolution que prennent les modes de recrutement dans la fonction publique ${ }^{52}$. La nécessité de faire évoluer le recrutement par concours dans la fonction publique est admise aujourd'hui comme une évidence. Le Rapport annuel sur la fonction publique 2010-2011 fait état d'une baisse notable des recrutements par la voie des concours externes ${ }^{53}$. Les concours d'entrée dans la fonction publique ne seraient pas encore suffisamment adaptés aux évolutions de l'emploi public.

\section{Des adaptations complexes voire inadaptées}

La modernisation des concours, notamment externes, consiste à faire passer des épreuves à des candidats qui pour la plupart n'ont aucune expérience professionnelle à proprement parler ou dont l'expérience reste de l'ordre du stage. Les candidats au concours externe sont majoritairement, et par définition, des candidats issus de la formation initiale. N'y a-t-il pas un paradoxe à demander à ces candidats un savoir-faire professionnel qu'ils n'ont pas pu encore acquérir ? Ou cela ne suppose-t-il pas que l'on s'intéresse préalablement à la formation des futurs candidats aux concours de la fonction publique en essayant de mettre en place des procédés pédagogiques leur permettant d'acquérir les réflexes professionnels et les compétences requises plutôt qu'un savoir strictement, ou presque, académique ? Ces questions sont en réalités anciennes et récurrentes en matière de fonction publique. Elles supposent que l'on tranche la délicate question du choix préférable entre un futur fonctionnaire généraliste ou un futur fonctionnaire technicien, spécialiste de l'administration. Ne doit-on pas considérer que tout candidat dont les connaissances sont générales et riches, notamment en culture générale, en droit, en histoire est susceptible de devenir une fois en poste un bon technicien de l'administration ? Sans compter que les compétences et autres aptitudes aujourd'hui demandées aux candidats aux

50. Ibid., p. 120 .

51. Ibid., p. 122.

52. Voir en ce sens, le contenu des épreuves du concours des Instituts régionaux d'administration : Arrêté du 6 juin 2008 fixant la nature, la durée et le programme des épreuves des concours d'entrée aux instituts régionaux d'administration, JORF, 20 juin 2008, p. 59.

53. p. 161. 
concours de la fonction publique ne font pas l'objet d'un traitement identique dans tous les établissements d'enseignement supérieur et dans toutes les préparations aux concours. Une formation pluridisciplinaire ne répond-elle pas mieux aux objectifs, aux valeurs et à l'éthique du service public qu'une formation trop professionnelle ?

La question de la professionnalisation des concours est donc une question difficile à trancher parce qu'elle suppose la remise en cause du fonctionnement même de notre fonction publique. La spécialisation des fonctionnaires est plus adaptée au système de l'emploi. Une trop grande spécialisation et une professionnalisation des candidats à la fonction publique peuvent entraîner des carrières bloquées, des expériences professionnelles difficiles à valoriser. Or, il n'est pas dans l'air du temps d'être enfermé dans sa carrière mais il faut plutôt être mobile. Les personnels administratifs de l'éducation nationale sont aujourd'hui confrontés, par exemple, dans le cadre des concours internes à des épreuves pour lesquelles ils n'ont jamais eu de formation. La demande est donc forte du côté de ce type de candidats pour procéder à une mise à niveau, non seulement dans les disciplines académiques mais également dans le cadre de préparation d'épreuves de reconnaissance des acquis de l'expérience professionnelle ou de préparation à l'entretien professionnel. Certains éléments de la modernisation des modes de recrutement sont plus faciles à mettre en œuvre que celui de la réforme des contenus des épreuves. Ainsi, les ministères sont-ils parvenus progressivement à une mutualisation de l'organisation des concours. Autrement dit, la réforme des aspects matériels des modes de recrutement pose moins de difficulté que celle des critères de recrutement.

Le concours reste, comme le remarque Jean-Michel Eymeri-Douzans, malgré ses «psychorigidités » administratives et juridiques, le meilleur outil permettant l'accès à la fonction publique, la promotion par le concours interne permet d'éviter une trop grande part d'arbitraire et une meilleure adéquation avec les valeurs du service public. La réforme des concours de la fonction publique constitue un véritable chantier auquel il convient de travailler collectivement sans perdre de vue les missions essentielles du service public et de la fonction publique. 